Plastic injection molding is widely used in many industrial applications. Plastic products are mostly used as disposable parts or as portable parts for fast replacements in many devices and machines. However, mass production is always adopted as an ideal method to cover the huge demands and customers' needs. The problems of warpage due to thermal stresses, non-uniform pressure distribution around cavities, shrinkage, sticking and overall products quality are some of the important challenges. The main objective of this work is to analyze the stress distribution around the cavities during the molding and demolding to avoid their effects on the product quality. Moreover, diagnosing the critical pressure points around and overall the cavity projection area, which is subjected to high pressure will help to determine the optimum pressure distribution and ensure filling all cavities at the same time, which is another significant objective. Computer-aided design (CAD) and CATIA V5R20 are adopted for design and modeling procedures. The computer-aided engineering (CAE) commercial software ABAQUS 6141 has been dedicated as finite element simulation packages for the analysis of this process. Simulation results show that stress distribution over the cavities depends on both pressure and temperature gradient over the contact surfaces and can be considered as the main affecting factor. The acceptable ranges of the cavity stresses were determined according to the following values: the cavity and core region temperature of $55-65^{\circ} \mathrm{C}$, filling time of 10-20 s, ejection pressure $0.85 \%$ of injection pressure, and holding time of 10-15s. Also, theoretical results reveal that the uniform pressure and temperature distribution can be controlled by adjusting the cavities layout, runner, and gate size. Moreover, the simulation process shows that it is possible to facilitate and identify many difficulties during the process and modify the prototype to evaluate the overall manufacturability before further investing in tooling. Furthermore, it is also concluded that tooling iterations will be minimized according to the design of the selected process

Keywords: mass production, injection, pressure, temperature, finite element, quality

\section{ANALYSIS OF THE MAIN FACTORS AFFECTING MASS PRODUCTION IN THE PLASTIC MOLDING PROCESS BY USING THE FINITE ELEMENT METHOD}

\author{
Hani Mizhir Magid \\ Corresponding author \\ $\mathrm{PhD}$ \\ Department Coordinator \\ Department of Power Mechanics \\ Technical College Al-Musaib - Babylon* \\ E-mail: han.magid@atu.edu.iq \\ Badr Kamoon Dabis \\ Master of Science \\ Department of Mechanical Engineering \\ Technical College Al-Musaib - Babylon* \\ Mohammad abed alabas Siba \\ Assistant Professor \\ Department of Mechanical Techniques \\ Institute of Technology - Baghdad \\ Middle Technical University \\ Alzafaranya, Baghdad, Iraq, 10074 \\ *Al-Furat Al-Awsat Technical University \\ Najaf, Kofa, Iraq, 54003
}

Received date 19.10.2021 Accepted date 10.12.2021 Published date 29.12.2021
How to Cite: Magid, H. M., Dabis, B. K., abed alabas Siba, M. (2021). Analysis of the main factors affecting mass production in the plastic molding process by using the finite element method. Eastern-European Journal of Enterprise Technologies, 6 (1 (114)), $65-71$. doi: https://doi.org/10.15587/1729-4061.2021.248375

\section{Introduction}

Mass production molds are normally comprised of many fabricated tooling inserts from different types of tool steel, which are assembled together to form the mold. The alignment and positioning of all mold elements require a high level of skills and accuracy. Due to a large number of cavities, the downstream problems between these cavities should be prevented to reduce the scrap levels and increase the consistency of the parts. Cavity pressure is considered as the main indicator for the whole process variation. Avoiding the problems of residual stresses, warpage problems and shrinkage are widespread by using numerical simulation. Moreover, theoretical predictions through calculating the pressure, temperature, and thermal stress distributions for injection molding by simulation are very important.

Model estimation of the molding process with analysis and simulation will allow for accurate results. It is always advised to apply the finite element method for this approach. The main advantages of the finite element method are the approximate convergence of solutions and enhanced accuracy of the result. The task of calculating the molten polymer behavior is greatly complicated by the need to model the physically nonlinear characteristics of the material flow. Moreover, the task of modeling a large number of cavities requires a large number of meshing elements and then the cost of calculations will increase significantly.

Therefore, studies that are devoted to this direction deal with the problem under consideration from different views. Most of these studies are considered irrelevant to the stated problem due to the large model used here. The majority of these results are based on the physical behavior of the molten polymer inside the mold cavities. Also, the methods that seek to determine the process constants will lead to large numbers of iterations, which increase the cost rapidly. 


\section{Literature review and problem statement}

Plastic parts are always produced by mass manufacturing through injection molding in many shapes and different sizes. In [1], the works were implemented on the low-volume manufacturing and effective cost prototyping method in injection molding. The paper [2] found that in polymers, the thermal expansion coefficient is varied directly with temperature, and that means this coefficient significantly increases with temperature, which will lead to drastic expansion of all mold parts and finally to mold failure. Employing rapid prototyping in injection molding reduces time by over $50 \%$ based on the rapid prototyping method. However, in terms of some considerations, the mass molding method has some limitations in terms of mold life, accuracy, and surface finish.

The paper [3] suggested that one of the most costly and time-consuming phases in the new product development is the creation of production components and prototyping production tools. When temperature distribution is homogeneous, the cycle time from processing to demolding will be less and the production rates will be higher. It is found that in mass tooling for injection molding, a non-optimal pressure distribution will result in warping and a non-acceptable product. Manufacturing and prototyping processes are developed rapidly in order to reduce the cost, manufacturing, and assembly time. More time and cost can be saved by using the tooling production process.

In [4], the effectiveness of creating complex shapes and geometries is one of the main challenges of using rapid manufacturing in the fabrication process of injection tooling. Nowadays, optimization processes based on finite-element methods are widely used in improving complex designs especially in injection tooling to increase the overall accuracy and performance. Results confirmed that by the simulation process, it is possible to predict and minimize many defects. Nevertheless, some unresolved issues, including time estimation and forecasting the behavior and characteristics of the overall injection process need to be addressed.

In [5], it is confirmed that computer simulation is an advanced tool to avoid any re-molding and reduce the cost of prototyping parts and minimize the time spent. The simulation process can help in re-modeling the parts many times and explore any possibilities for building the geometry, size, and distribution layout besides testing and verifying many times in the injection molding process. It was found in [6] that the less tool manufacturing process is the main advantage in both rapid prototyping and rapid manufacturing technologies. In the environment of concurrent engineering, the approach of cycle product development, manufacturing considerations, and design process are the main factors that united together in terms of product quality.

The paper [7] uses the finite element to analyze and estimate the main factors, which particularly affect pressure and temperature distribution. The paper [8] shows that the combination of runner size with cavity size and some other selected factors are very helpful in estimating the filling performance during the injection process. Consequently, the advantage of mass injection molding is its suitability to obey customer demands with high efficiency. The downside of this method is that it is apt in estimating the filling of small molds.

Innovative manufacturing processes are always associated with many challenges especially product complexity. The recent trend of mass production has many advancements to overcome these challenges. To build up a new and optimized strategy for the manufacturing process, integrating between many factors is very essential. The paper $[9,10]$ shows that these factors include material, production, process, and information with computer-aided design techniques. The new trends in the efforts of improving product quality focus on the design process for complex molding. The problems of high requirements like hardened tool steel and long lead times for the conventional molding process can be replaced over a large number of insert cavities, which can be replaced and changed at any time.

Nowadays, competition in a mold-making industry is expanded due to high demands and commercial opportunities. Mass production by using the method of mass tooling involved with mold insert provided the alternative and effective way and offers better accuracy. Some shortcomings like alignment, pressure distribution need to be addressed as explained in [11].

In [12], the warpage deformation results from simulation show that the analysis method is so useful in injection molding and can cover and diagnose the main manufacturing shortage before investment in tooling. The data obtained in [13] show that the product is exposed to different mechanical and thermal conditions during the removal and ejection from the cavity. In general, the generation of flow stresses and thermal stresses will lead to either damage to the product surface or detrimental some dimension and also may result in cracking.

The work [14] clarifies that limitations and potential in using simulation and optimization in mass production molding should be used to help in avoiding the main obstacles and errors before tooling investing. The work [15] notes that the finite element analysis can be considered a great tool for prediction and estimation in the molding process. The analysis procedure involves spitting the part in finite element by using the mesh option. Then applying different boundary conditions and mathematical constraints and finding the suitable interaction between these elements. This criterion will improve the accuracy and overall findings. However, these boundary conditions should be modified according to the layout nature and cavities distributions.

The work [16] shows that designing a large-size mold in mass production involves high necessary costs due to using some accuracy and complex machines like CNC. Manufacturing of sophisticated mold tools like inserts, cavities will impose extra constraints on the market due to customers' needs. In [17], it is found that process parameters like cavity pressure and melt temperature significantly affect the specific volume of the part. For this purpose, and to decrease warpage, it is necessary to create a dynamic mold for temperature control.

The main reason for this research is to find out and diagnose the concentration and the effects of stresses and high-pressure points inside the cavities by simulation to avoid the deformation under dynamic pressure. The analytical solution is less time-consuming compared to physical experiments. This target was achieved in some literature here, but others do not. Consequently, conducting this study to build the model is an important target in this field.

In the paper, efficient and precise modeling for the part and mold according to the design layout has been implemented. Then, the simulation and analysis were used for estimating the critical zones and weak points. In the next step, the factors of the injection process, such as temperature, pressure, and stresses affecting the product quality were analyzed and refined, and buildup according to the downside of researchers works. Finally, some points are presented as conclusions and recommendations for this work. 


\section{The aim and objectives of the study}

The aim of the study is to build a finite element model for injection molding, which contains many cavities for the purpose of mass production. The simulation findings could be used to determine and investigate the interaction between some different parameters during this dynamic process, which can help manufacturers to avoid and overcome many fabrication errors.

To achieve this aim, the following objectives are accomplished:

- to clarify and estimate the problems and effects of stress generation, pressure distribution inside the cavities and predict the weak points in injection molding before building the actual tools;

- to explore the general solutions of filling and ejection (demolding) for different pressure points in multi-cavity molding. cess before manufacturing and assembly can prevent and avoid a lot of mistakes and save both time and cost. The mold plate, which contains the cavities and inserts is designed and modeled according to the pressure and temperature calculations. The layout should be set and positioned to ensure the equilibrium of pressure and temperature at all points. Fig. 3 illustrates this layout.

Fig. 4 illustrates the modeling process of the mold during the end of the injection process and all parts are fully completed.

Demolding and ejection of the parts from cavities without any deformation during the cycle time and before the complete cooling are considered an essential step. Modeling this step will help to avoid any interference between the ejectors and cavity inserts and prevent any misalignments between the parts. Fig. 5 below illustrates the modeling process of the ejection of the part at the end of the injection process to remove the parts safely.

\section{Materials and methods}

The main important idea in this research is how to maximize the production of plastic products besides quality assurance via controlling some influencing factors. The first step is to select the layout of the plastic product under study with all dimensions as in Fig. 1, $a, b$ below.

The design procedure for injection molding includes designing all mold parts according to the standard. Mold design includes distribution of the cavities over the effective surface area of the molding plate in a harmonic manner that allows easy manufacturing and assembly. Fig. 2 shows the design of the mold assembly and cavities distribution with all important dimensions.

In the above design, the mold contains 20 cavities in the same mold plate. The distribution of these cavities should be calculated with high accuracy to keep homogenous pressure and temperature balanced. The runner dimension and layout are important as a part of the feeding system to ensure the filling and temperature balance. Polyvinyl chloride (PVC) is used in the test. Shrinkage of this plastic material is $2 \%$ with a density of $1.3-1.7 \mathrm{~g} / \mathrm{cm}^{3}$ and $212{ }^{\circ} \mathrm{C}$ melting temperature.

However, modeling the overall process is more essential in this type of manufacturing process. Through modeling techniques, it is possible to avoid many errors and mistakes before any further actions. Interference between some dimensions during mold assembly always happens. Modeling the overall pro-
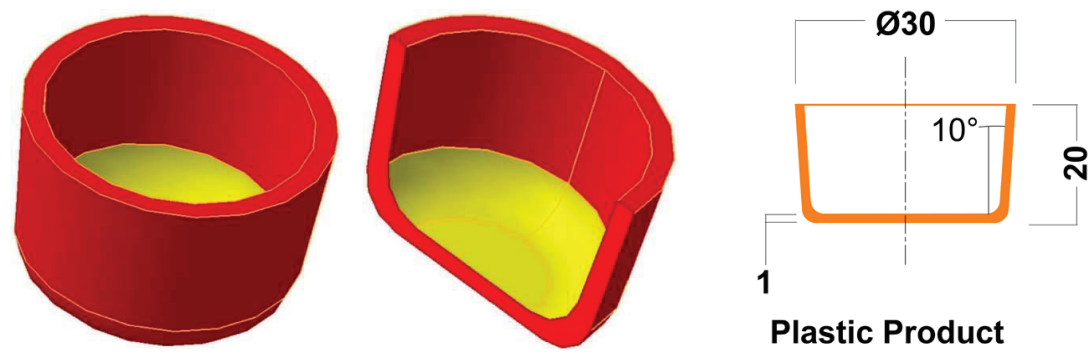

Plastic Product

$a$

$b$

Fig. 1. Product geometry: $a-$ in three dimensions; $b-$ in two dimensions

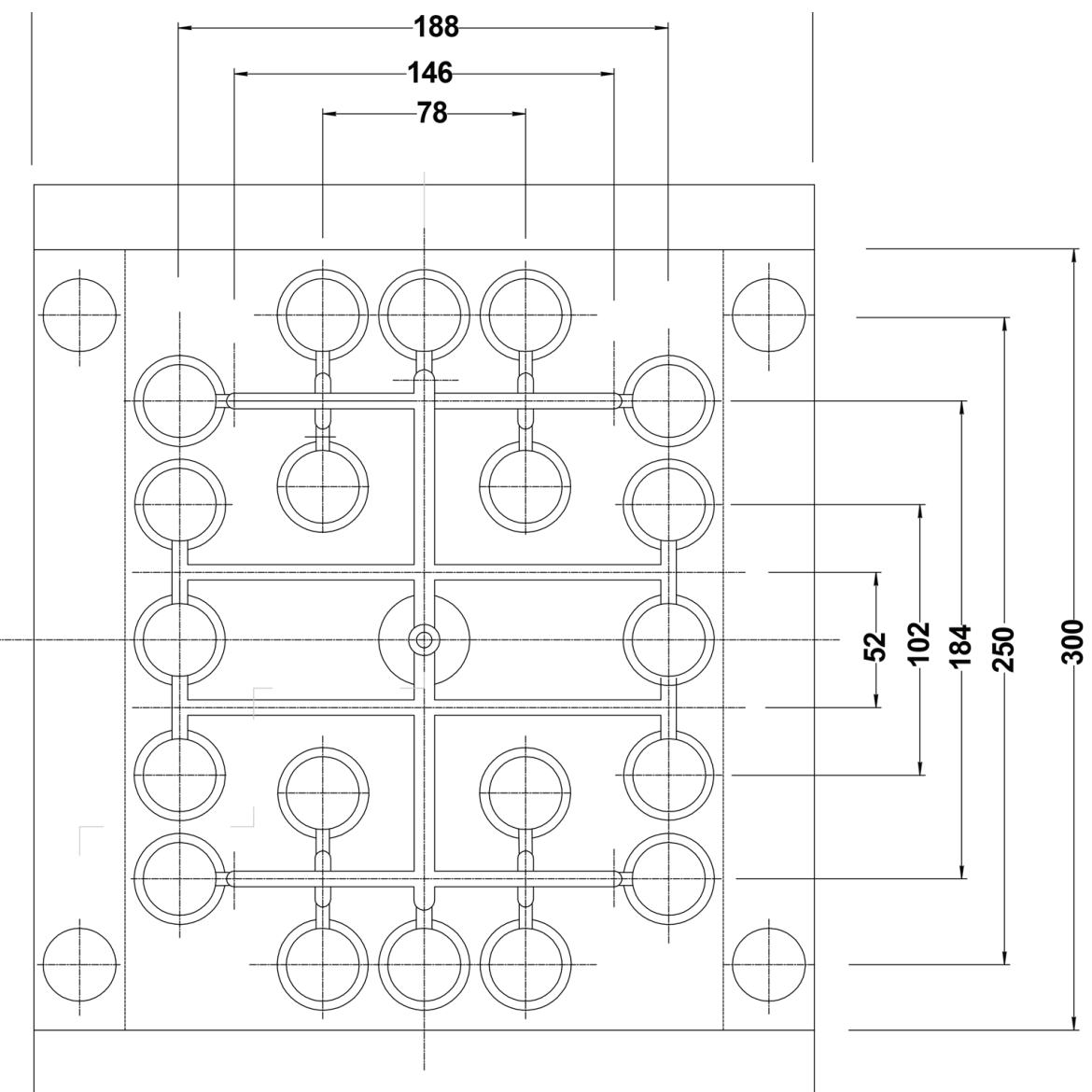

Fig. 2. Mold plate including 20 cavities distribution 


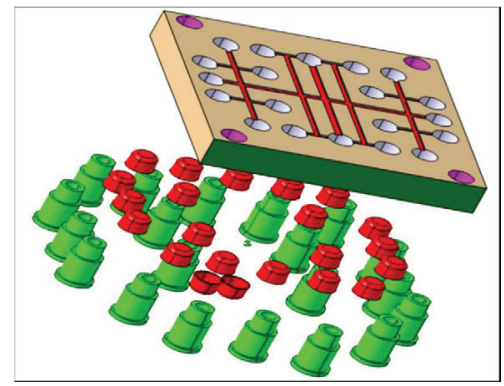

Fig. 3. Modeling of mold plate with cavities and products

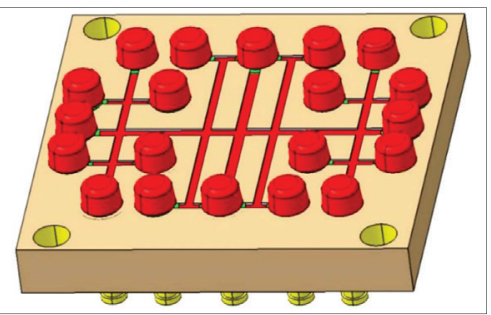

Fig. 4. Modeling of the mold illustrates the filled cavities with runner and gates

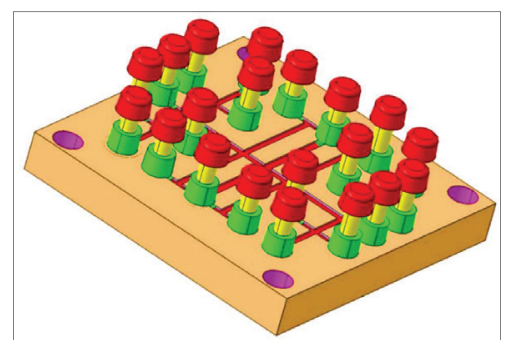

Fig. 5. Modeling of demolding 20 parts from the cavities after full curing

The simulation process was implemented by ABAQUS/ (CAE) following the standard criteria in which the user must follow some sequence steps. The main important simulation steps are summarized as a flowchart in Fig. 6 below.

Simulation of this type of manufacturing process aims to enhance the understanding and find the visibility of prototyping the mass production in plastic molding and explore the best solutions to overcome many problems happening during this process like thermal stresses and non-homogeneous pressure distribution. Different boundary conditions are used in this analysis according to the step functions. Displacement rotation is the important boundary condition used in the first step, which includes constraining the mold plate from any movement and rotation in all directions and applying the pressure load to eject the product in the final step. Fig. 7 below illustrates this boundary condition.

The relation between the contact surfaces is considered as master and slave. The insert surface and ejector are considered as master, while the product is considered as slave surface. Interaction property between these parts in the second step is used to be surface to surface contact property with sliding behavior, as shown in Fig. 8.

Seeding and meshing the parts is an essential step before any further action. Depending on the nature of element type, mesh control varies according to the part geometry. Sweep, medial axis, and structural mesh with quad and quad dominated element shape are used to implement meshing of all the parts individually.

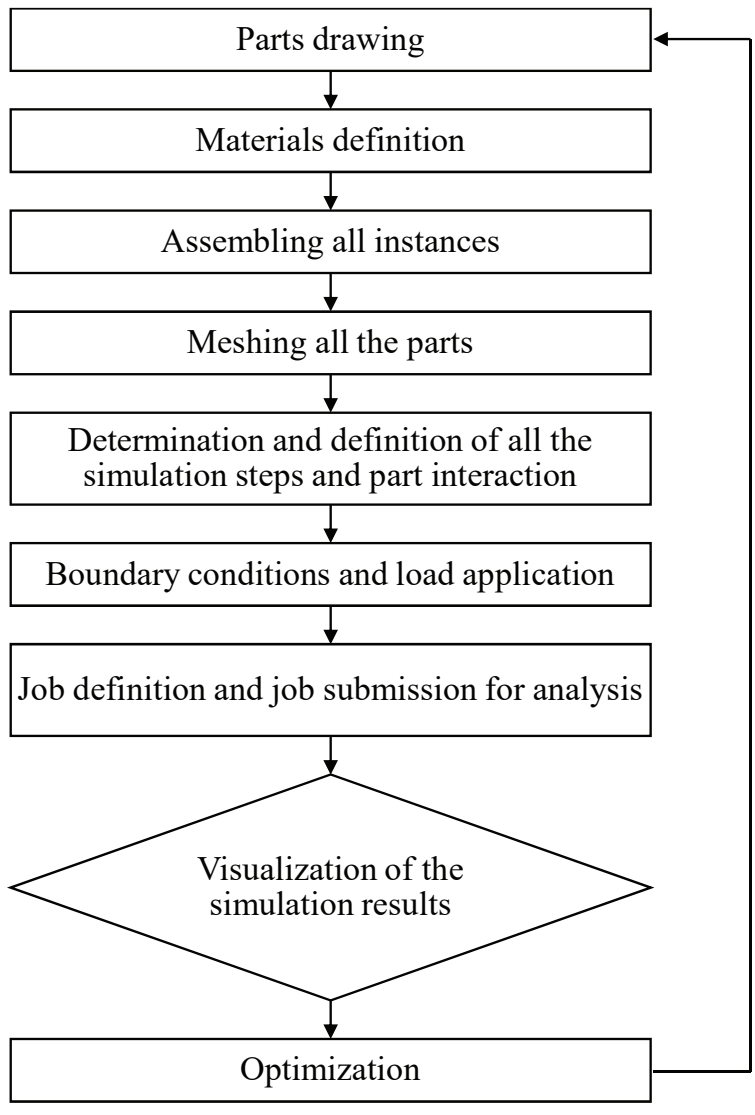

Fig. 6. Simulation flowchart in ABAQUS/(CAE)

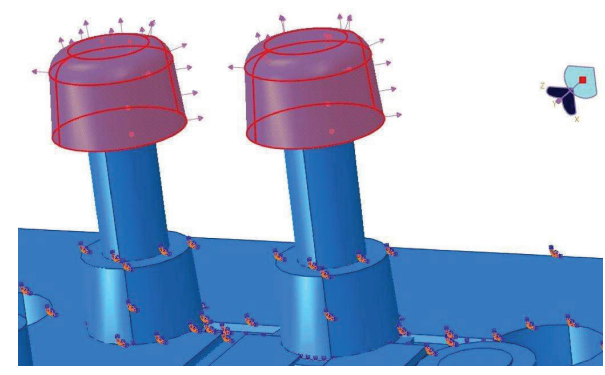

Fig. 7. Boundary conditions include cavity constrain and applied load

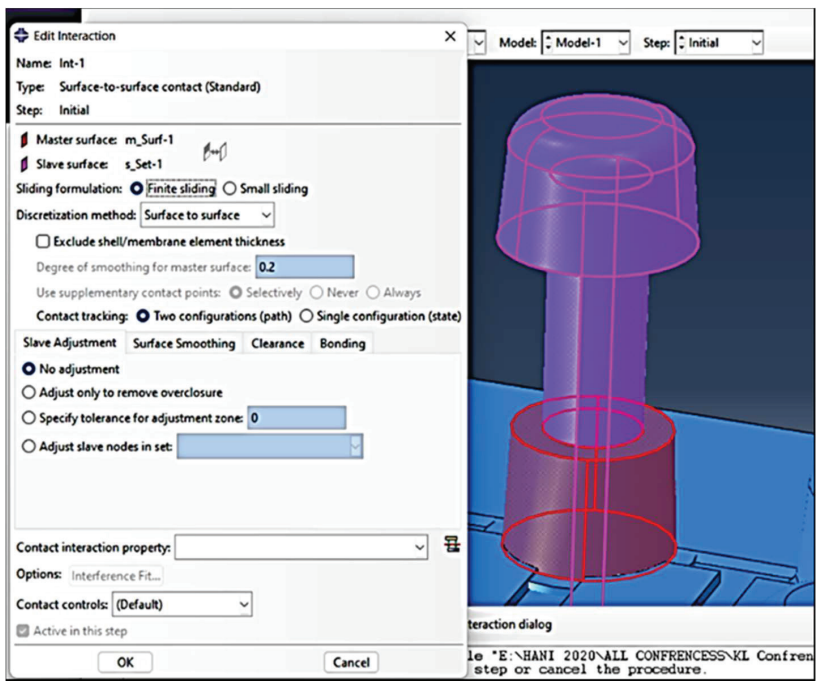

Fig. 8. Sliding interaction behavior between the parts 
Fig. 9 illustrates the meshed assembly according to the standard mesh rules used in ABAQUS/(CAE).

In this research, the analysis focuses on the weak points around the cavity area, which are subjected to high pressure and temperature and result in high-stress concentration (Fig. 10).

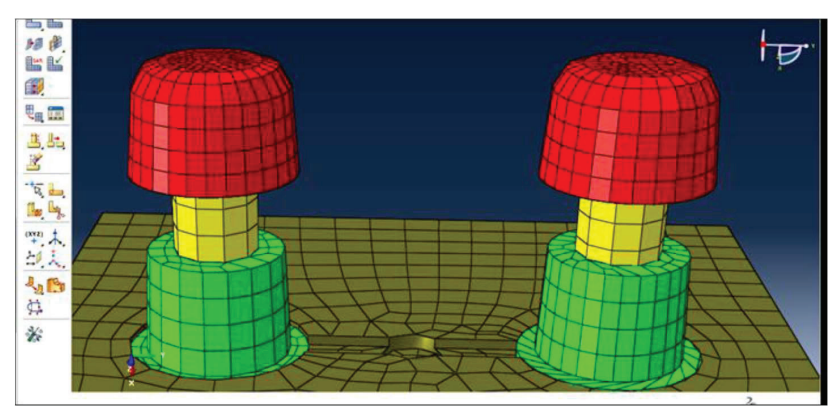

Fig. 9. Medial axis and structural mesh for the inserts, ejector, and product

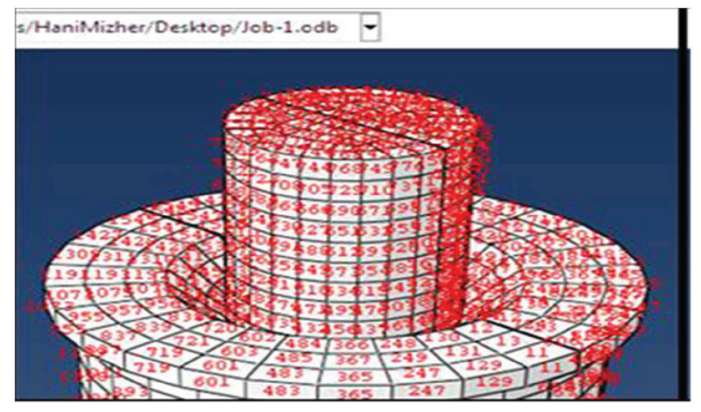

Fig. 10. Elements label including numbering all elements of ejector and cavity

Consequently, the first important step leading to accurate results is to label all the important elements, which enables us to determine the position of each element exactly as shown in Fig. 10.

In this analysis, stress distribution, ejection pressure, and pressure drop inside and around the cavities layout have been discussed. The analysis has been carried out for a total of 20 cavities using ABAQUS/CAE 6141 software.

\section{Results of analysis and simulation of injection} parameters and mold layout

\section{1. Pressure distribution around cavities}

The molten injected polymer will apply high pressure around the cavity surface, and this pressure may cause non-symmetric product dimensions. The contour plot of pressure distribution around the cavity shown in Fig. 11 revealed that the pressure concentration in contact surfaces between the cavity and the product (the master and slave) and inside the cavity will be at maximum values. Due to the interaction between the contact surfaces and high sticking forces during the demolding action will be maximized due to a decrease in contact surface area.

The variation in cavity pressure with injection time is normally due to many reasons like product thickness, the distance of cavity from the injection gate, and material viscosity. Material viscosity is normally affected by a high-temperature gradient and this will lead to an increase in the cavity pressure with time. Fig. 12 shows that the cavity pressure will reach the maximum value in a short period due to fast hold- ing and hot molten polymer and then drop to the minimum value after the clamping is released.

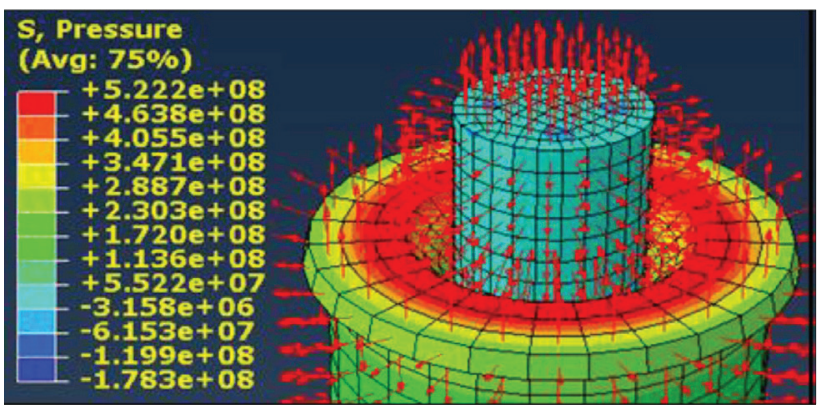

Fig. 11. Contour of pressure distribution around the cavity

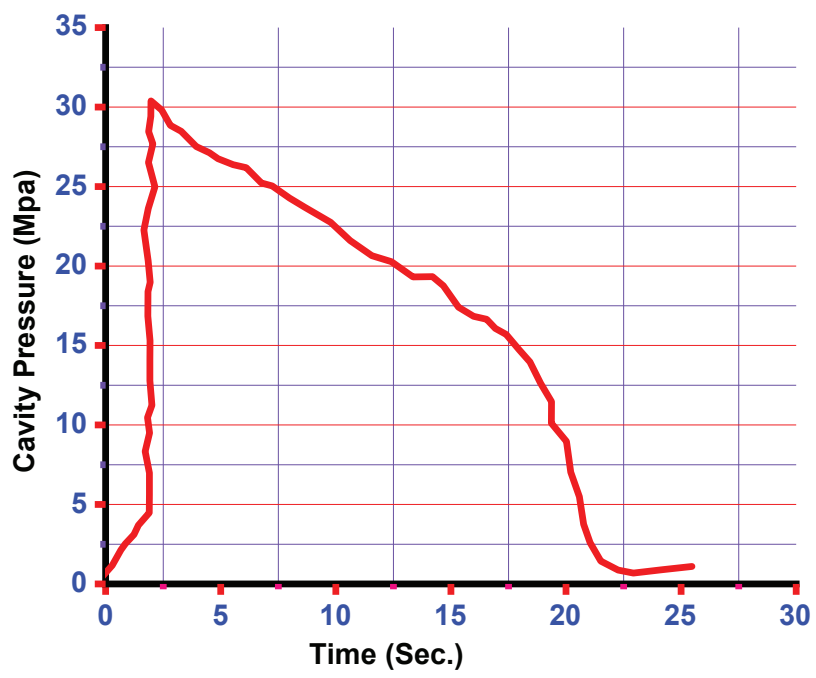

Fig. 12. Variation in cavity pressure with injection time

The pressure variation between cavities normally happens in this type of molding due to a large number of cavities, and also due to the distance between the sprue bush and cavity centers.

\section{2. Stresses distribution}

With a continuous injection process, the cavity temperature will rapidly increase to the maximum elevated value. Consequently, stresses will generate and build up around and along the cavity surface area. The contour plot of the average principal stresses distribution shown in Fig. 13 illustrates that the contact zone between the two sides of the mold is less thermally stressed than the inside cavity due to the cooling cycle, while the inside cavity including the ejectors is highly stressed. Stresses are concentrated around the cavity and ejector area due to two reasons. The first one is due to the property of highly sticking between product and ejector, and the second is due to the shrinkage problems, which depend on the material type, cooling rate, and draft angle. This problem needs to be avoided by adjusting the temperature and injection pressure by selecting suitable runner and gate dimensions.

During the injection process, and when the mold temperature is low, the molten polymer in contact with the cavity will rapidly be cooled in a short time and this may lead to some surface defects. Moreover, at high-temperature levels, the cavity will be filled in a short time due to a decrease in resistance to the molten polymer then the mold cooling will 
prolong more time, but at the same time, the internal stresses will buildup rapidly. Fig. 14 below illustrates the relation between cavity temperature and stress generation.

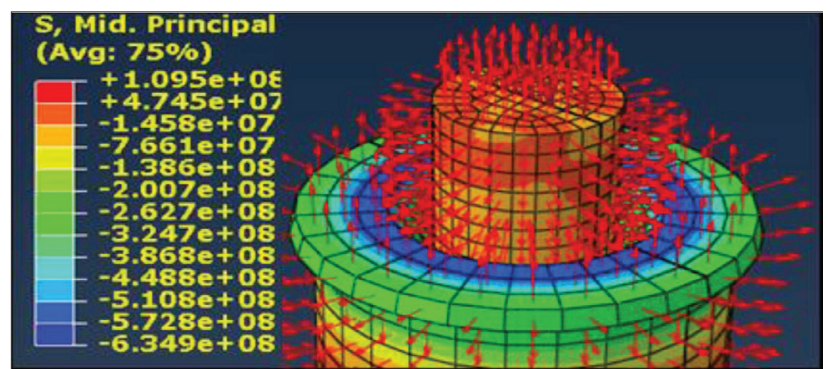

Fig. 13. Contour of principle stresses around the cavity and ejector area

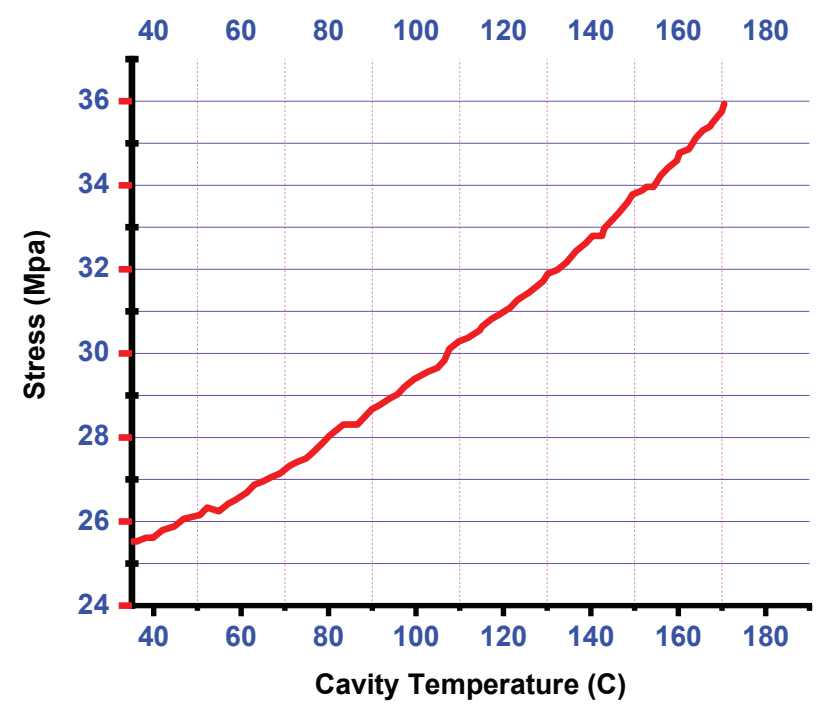

Fig. 14. Relation between cavity temperature and stress generation

Ejection force is directly proportional to holding time and inversely proportional to holding pressure. Fig. 15 illustrates that the ejection force will raise at the beginning of ejection time due to the large contact surface area between ejectors and mold part. But ejection pressure starts to drop down with time due to a decrease in contact force between the parts.

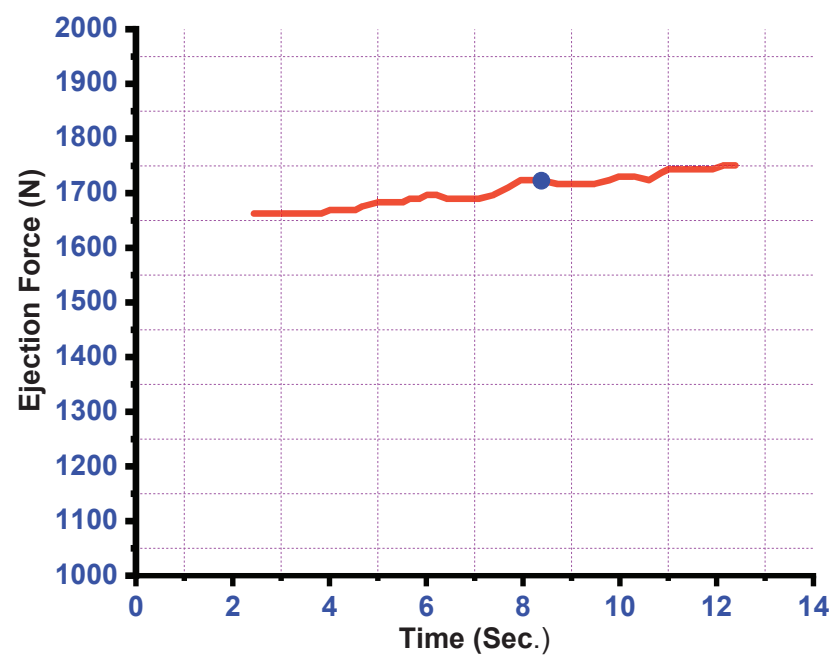

Fig. 15. Relationship between time and ejection force he above profile will be subject to change if some dependent variables like flow rate, temperature, time, and holding pressure change. Also, the shrinkage percentage of the injected material highly affects the value of ejection force.

\section{Discussion of the results of analysis and simulation of injection molding}

The results obtained with the proposed method are intended to show the weak points in this molding type and focus on stress and pressure distribution inside many cavities in order to suggest the homogeneous distribution by comparing with some of the existing researches.

We have considered reducing all types of intensive loads along the contact surfaces to avoid product distortion.

Consequently, according to the pressure and temperature distribution on the contact surfaces, the relationship between the cavity pressure thermal stresses has been determined.

Based on the simulation results, the influence of stresses has been determined under different types of boundaries by considering that all inserts and cavities (non-deformable parts) as masters and products (deformable parts) as a slave.

To find out a suitable result, the findings of previous research have been stated for evaluation purposes. The mold under study consists of many materials with different mechanical and physical properties. These materials are defined as well and assigned to their sections based on specific criteria used in the software. Due to the sliding interaction behavior between the parts (master and slave) as mentioned in Fig. 8, and the nature of constraints as in the boundary condition (Fig. 7), the displacement of the stochastic elements (deformation) can be eliminated to the minimum. Consequently, the high pressure and highly stressed zones over the mold surface area are concentrated with their maximum values in the non-deformable parts (cavities and inserts) as illustrated in Fig. 11 and Fig. 13.

The medial axis and structural mesh method used in seeding and meshing the master parts (inserts, cavities, and ejector) as in Fig. 9, and associated this mesh with relative geometries will ensure a uniform elements displacement during the product ejection step.

This will ensure a little variation in the cycle time of product demolding as in Fig. 15. It is important to reveal that there are some limitations regarding the nature of the molten polymers used and the percentage of shrinkage. The nonlinearity and material orientation inside. The cavity imposes some restrictions on the accuracy of parameters calculation.

The main limitations of this study involve some boundary conditions related to some necessary restrictions of parts due to high numbers of cavities and inserts.

The disadvantage of handling this analysis method is the need for a large amount of data and it is always time-consuming due to running and repeating the simulation many times till finding accurate results. Failure in determining the exact formulas and boundary conditions can affect the results significantly.

The development of such research types is related to overcoming and eliminating some of the shortcomings and restrictions, which lead to raising the concentration and values of stresses by using the suitable boundary conditions and interaction types between the contact parts. To eliminate the generation of stresses, which result in high and non-homogeneous pressure distribution around and inside the cavities, it is recommended to partition the simulated part in different zones to increase the element density, which leads to the best accuracy. 


\section{Conclusions}

1. Simulation results predict the concentration and distribution of thermal stresses around the specific areas, temperature variations, and pressure distribution over the mold plate area. Due to the large mold area, which contains many cavities, it is found that the temperature gradients over the mold will vary from one cavity to another. Simulation results revealed that the variation range between the near and far cavities from the injection center is approximately $2{ }^{\circ} \mathrm{C}$. Also, the temperature values in the core and cavity zone, which lead to acceptable ranges of the cavity stresses are in the range of $55-65{ }^{\circ} \mathrm{C}$ with a filling time of $10-20 \mathrm{~s}$. This can be accepted due to the large surface area and cooling difference. Also, the thermal stresses are always concentrated over the interaction and contact surfaces like ejectors, inner cavities, and cores.

2. The ejection system needs to work systematically and the ejection pressure should be uniform over cavities to avoid any warpage in products during the demolding time. Heating the mold before the process and choosing a suitable runner geometry are some of the suggested solutions to solve some problems like shortage filling, which normally occurs due to the pressure difference. One of the important conclusions is related to the relationship between cavity pressure and ejection force. The required force to remove the parts during ejection will increase rapidly due to high shrinkage and high sticking force with a cavity. It is important to adjust the injection system like a runner, gates, pressure, and cooling system to avoid these shortcomings. It is concluded that the percentage of ejection pressure is $0.85 \%$ of injection pressure value, and the value of the holding time is $10-15 \mathrm{~s}$.

\section{Acknowledgments}

The authors introduce their thanks to all friends and colleagues who support us to achieve this research paper. Also, all authors of this paper are offering best wishes to the editorial board of this esteemed journal.

\section{References}

1. Bagalkot, A., Pons, D., Symons, D., Clucas, D. (2019). Categorization of Failures in Polymer Rapid Tools Used for Injection Molding. Processes, 7 (1), 17. doi: https://doi.org/10.3390/pr7010017

2. Nagahanumaiah, Subburaj, K., Ravi, B. (2008). Computer aided rapid tooling process selection and manufacturability evaluation for injection mold development. Computers in Industry, 59 (2-3), 262-276. doi: https://doi.org/10.1016/j.compind.2007.06.021

3. Gries, S., Meyer, G., Wonisch, A., Jakobi, R., Mittelstedt, C. (2021). Towards Enhancing the Potential of Injection Molding Tools through Optimized Close-Contour Cooling and Additive Manufacturing. Materials, 14 (12), 3434. doi: https://doi.org/10.3390/ma14123434

4. Hartono, M., Pratikto, Santoso, P. B., Sugiono (2020). Optimization on the Injection Molding Propypopylene Parameters Using Central Composite Design for Minimizing Defects. Journal of Southwest Jiaotong University, 55 (2). doi: https://doi.org/10.35741/ issn.0258-2724.55.2.42

5. Kriesi, C., Bjelland, Ø., Steinert, M. (2018). Fast and iterative prototyping for injection molding - a case study of rapidly prototyping. Procedia Manufacturing, 21, 205-212. doi: https://doi.org/10.1016/j.promfg.2018.02.112

6. Mansour, S., Hague, R. (2003). Impact of rapid manufacturing on design for manufacture for injection moulding. Proceedings of the Institution of Mechanical Engineers, Part B: Journal of Engineering Manufacture, 217 (4), 453-461. doi: https://doi.org/ 10.1243/095440503321628134

7. Liang, W., Ge, X., Ge, J., Li, T., Zhao, T., Chen, X. et. al. (2018). Reduced Graphene Oxide Embedded with MQ Silicone Resin NanoAggregates for Silicone Rubber Composites with Enhanced Thermal Conductivity and Mechanical Performance. Polymers, 10 (11), 1254. doi: https://doi.org/10.3390/polym10111254

8. Kazmer, D. O., Speight, R. G. (1997). Polymer Injection molding Technology for the Next Millennium. Journal of Injection Molding Technology, 1 (2), 81-90. Available at: https://www.researchgate.net/publication/237673663_Polymer_Injection_Molding_ Technology_for_the_Next_Millennium

9. Abdulhameed, O., Al-Ahmari, A., Ameen, W., Mian, S. H. (2019). Additive manufacturing: Challenges, trends, and applications. Advances in Mechanical Engineering, 11 (2), 168781401882288. doi: https://doi.org/10.1177/1687814018822880

10. Vieten, T., Stahl, D., Schilling, P., Civelek, F., Zimmermann, A. (2021). Feasibility Study of Soft Tooling Inserts for Injection Molding with Integrated Automated Slides. Micromachines, 12 (7), 730. doi: https://doi.org/10.3390/mi12070730

11. Hussin, R., Sharif, S., Nabiałek, M., Zamree Abd Rahim, S., Khushairi, M. T. M., Suhaimi, M. A. et. al. (2021). Hybrid Mold: Comparative Study of Rapid and Hard Tooling for Injection Molding Application Using Metal Epoxy Composite (MEC). Materials, 14 (3), 665. doi: https://doi.org/10.3390/ma14030665

12. Kim, H.-S., Lee, W.-G., Lee, C.-H., Lee, K. D. (2020). Optimization for the prepreg compression molding of notebook computer cover using design of experiment and finite element method. SN Applied Sciences, 2 (9). doi: https://doi.org/10.1007/s42452-020-03416-4

13. Zhou, H. (Ed.) (2013). Computer modeling for injection molding. Simulation, Optimization, and Control. John Wiley \& Sons, Inc.

14. Loaldi, D., Regi, F., Baruffi, F., Calaon, M., Quagliotti, D., Zhang, Y., Tosello, G. (2020). Experimental Validation of Injection Molding Simulations of 3D Microparts and Microstructured Components Using Virtual Design of Experiments and Multi-Scale Modeling. Micromachines, 11 (6), 614. doi: https://doi.org/10.3390/mi11060614

15. Polanco-Loria, M., Clausen, A. H., Berstad, T., Hopperstad, O. S. (2010). Constitutive model for thermoplastics with structural applications. International Journal of Impact Engineering, 37 (12), 1207-1219. doi: https://doi.org/10.1016/j.ijimpeng.2010.06.006

16. Hopmann, C., Xiao, C., Kahve, C. E., Fellerhoff, J. (2021). Prediction and validation of the specific volume for inline warpage control in injection molding. Polymer Testing, 104, 107393. doi: https://doi.org/10.1016/j.polymertesting.2021.107393 